\title{
Upper Limit on the QCD Axion Mass from Isolated Neutron Star Cooling
}

\author{
Malte Buschmann $\odot,{ }^{1}$ Christopher Dessert $\odot,{ }^{2,3,4}$ Joshua W. Foster $\odot,{ }^{5}$ Andrew J. Long $\odot,{ }^{6}$ and Benjamin R. Safdi $\odot^{3,4}$ \\ ${ }^{1}$ Department of Physics, Princeton University, Princeton, New Jersey 08544, USA \\ ${ }^{2}$ Leinweber Center for Theoretical Physics, Department of Physics, University of Michigan, Ann Arbor, Michigan 48109 USA \\ ${ }^{3}$ Berkeley Center for Theoretical Physics, University of California, Berkeley, California 94720, USA \\ ${ }^{4}$ Theoretical Physics Group, Lawrence Berkeley National Laboratory, Berkeley, California 94720, USA \\ ${ }^{5}$ Center for Theoretical Physics, Massachusetts Institute of Technology, Cambridge, Massachusetts 02139, USA \\ ${ }^{6}$ Department of Physics and Astronomy, Rice University, Houston, Texas 77005, USA
}

(Received 1 December 2021; accepted 2 February 2022; published 1 March 2022)

\begin{abstract}
The quantum chromodynamics (QCD) axion may modify the cooling rates of neutron stars (NSs). The axions are produced within the NS cores from nucleon bremsstrahlung and, when the nucleons are in superfluid states, Cooper pair breaking and formation processes. We show that four of the nearby isolated magnificent seven NSs along with PSR J0659 are prime candidates for axion cooling studies because they are coeval, with ages of a few hundred thousand years known from kinematic considerations, and they have well-measured surface luminosities. We compare these data to dedicated NS cooling simulations incorporating axions, profiling over uncertainties related to the equation of state, NS masses, surface compositions, and superfluidity. Our calculations of the axion and neutrino emissivities include highdensity suppression factors that also affect SN 1987A and previous NS cooling limits on axions. We find no evidence for axions in the isolated NS data, and within the context of the Kim-Shifman-VainshteinZakharov QCD axion model, we constrain $m_{a} \lesssim 16 \mathrm{meV}$ at $95 \%$ confidence level. An improved understanding of NS cooling and nucleon superfluidity could further improve these limits or lead to the discovery of the axion at weaker couplings.
\end{abstract}

DOI: 10.1103/PhysRevLett.128.091102

The quantum chromodynamics (QCD) axion is a wellmotivated beyond-the-standard-model particle candidate that may explain the absence of the neutron electric dipole moment [1-4] and the dark matter (DM) in our Universe [5-7]. However, the axion remains remarkably unconstrained experimentally and observationally, despite nearly 45 years of effort dedicated to axion searches (see Ref. [8] for a review). The QCD axion is primarily characterized by its decay constant $f_{a}$, which sets both its mass [9] $m_{a} \approx$ $5.7 \mu \mathrm{eV}\left(10^{12} \mathrm{GeV} / f_{a}\right)$ and its interaction strengths with matter. Requiring $f_{a}$ below the Planck scale implies $m_{a} \gtrsim 10^{-12} \mathrm{eV}$. The axion mass is currently bounded from above by supernova (SN) and stellar cooling constraints at the level of tens of meV, subject to model dependence and astrophysical uncertainties that are discussed further below.

The neutron star (NS) axion constraints presented in this Letter are part of a broader effort to probe the QCD axion over its full possible mass range. Black hole superradiance disfavors QCD axion masses $m_{a}<2 \times 10^{-11} \mathrm{eV} \mathrm{[10-12],}$

Published by the American Physical Society under the terms of the Creative Commons Attribution 4.0 International license. Further distribution of this work must maintain attribution to the author(s) and the published article's title, journal citation, and DOI. Funded by SCOAP ${ }^{3}$. while the ADMX experiment has reached sensitivity to Dine-Fischler-Srednicki-Zhitnitsky (DFSZ) $[13,14]$ QCD axion DM over the narrow mass range $m_{a} \sim 2.7-4.2 \mu \mathrm{eV}$ by using the axion-photon coupling [15-17]. Apart from these constraints, and additional narrow-band constraints from the ADMX [18] and HAYSTAC [19] experiments at the level of the more strongly coupled Kim-ShifmanVainshtein-Zakharov (KSVZ) [20,21] axion, there is nearly a decade of orders of magnitude of parameter space open for the axion mass that is unprobed at present. On the other hand, near-term plans exist to experimentally cover most of the remaining parameter space for QCD axion DM, including ABRACADABRA [22-24], DM-Radio [25], and CASPEr [26-28] at axion masses $m_{a} \ll \mu \mathrm{eV}$, ADMX and HAYSTAC at axion masses $m_{a} \sim 1-100 \mu \mathrm{eV}$, and MADMAX and plasma haloscopes at masses $\sim 40$ $400 \mu \mathrm{eV}[29,30]$. However, astrophysical searches such as that presented in this Letter play an important role in constraining higher axion masses near and above the meV scale. Axions with $m_{a} \gtrsim \mathrm{meV}$ are difficult to probe in the laboratory, even under the nontrivial assumption that the axion is DM (but see Ref. [31-36] for proposals). While it was previously thought that the QCD axion cannot explain the entirety of DM at masses at and above $\sim \mathrm{meV}$ masses, this assumption has been challenged, recently (see, e.g., Ref. $[37,38])$, further motivating the search for meV-scale axions. 


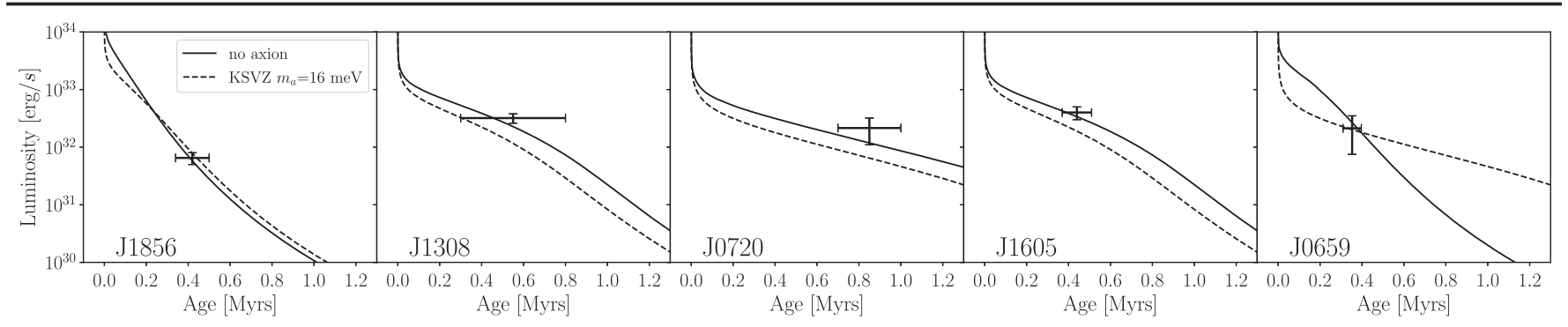

FIG. 1. The luminosity and age data for each of the NSs considered in this Letter (see Table I). We show the best-fit cooling curves computed in this Letter for each of these NSs under the null hypothesis and with the axion mass fixed to $m_{a}=16$ meV, which is our 95\% upper limit on the QCD axion mass in the context of the KSVZ model.

The fundamental idea behind how axions may modify NS cooling is that these particles, just like neutrinos [39], may be produced in thermal scattering processes within the NS cores and escape the stars due to their weak interactions $[40,41]$. Most previous studies of axion-induced NS cooling have focused on either proto-NSs, like that from SN 1987A [42-46], that are seconds old or young NSs like Cas A [47-52], which has an age 300 years. In this Letter, we show that robust and competitive constraints on $m_{a}$ may be found from analyses of older NS cooling, focusing on NSs with ages $\sim 10^{5}-10^{6}$ years (see Ref. [53,54] for related studies of older NSs). This is important when considering the possible issues that affect the SN 1987A and Cas A constraints, such as the lack of fully self-consistent 3D simulations [43] for SN 1987A and uncertainties related to the formation of the proto-NS [55] (but see Ref. [56]). Axion constraints from Cas A arise by using the observed temperature drop of the young NS over the past two decades by the Chandra telescope, but it was realized, recently, that this drop may be due to a systematic evolution of the energy calibration of the detector over time [57]. Moreover, the Cas A constraints are typically derived under the assumptions of specific superfluidity and equation of state (EOS) models, which are themselves uncertain. It is clear that additional, independent probes are needed to robustly disfavor or detect the QCD axion at masses above a few meV.

Isolated NS data and modeling.-In this Letter, we use luminosity and kinematic age data from four of the seven magnificent seven (M7) NSs, which are those where kinematic age data is available (see Table I and Fig. 1 for their relevant data). We add to this list PSR J0659, identified with the Monogem ring, as it also has an age above $10^{5}$ years known from kinematic considerations $[58,59]$ and a thermal luminosity measurement. The NSs with ages $\sim 10^{5}$ years live at a unique era, as illustrated in Fig. 2, where cooling from axion bremsstrahlung emission is maximally important; at lower ages neutrino emission plays a more important role since the neutrino (axion) emissivity scales as $\propto T^{8}\left(T^{6}\right)$ with temperature $T$, while at older ages the thermal surface emission dominates the energy loss. We discuss NSs with ages less than $10^{5}$ years, including Cas A, in the Supplemental Material (SM) [60]. The age data have been determined by tracing the NSs back to their birthplaces. A measured NS orbit is run backwards in the Galactic potential and a parent stellar cluster is identified in each case. J1856 and J1308 are found to originate in the Upper Scorpius OB association [102,103]. J0720 was likely born in the Trumpler association [104]. J1605 can be associated with a runaway former binary companion, which was disrupted in a supernova [105].

The thermal luminosity data for these NSs are measured from spectral fitting of NS surface models to the x-ray spectra. The strong magnetic fields create localized temperature inhomogeneities on the surfaces, so the total thermal luminosity is a more robust observable for our purposes, since it is less affected by the temperature inhomogeneities than direct temperature measurements. For this reason, we use the luminosity data in this Letter rather than surface temperature measurements [106]. Typically, a NS atmosphere model or a double-blackbody model is fit to the x-ray spectral data. For J1856, a thin partially ionized hydrogen atmosphere model suggests our lower luminosity bound $\sim 5 \times 10^{31} \mathrm{erg} / \mathrm{s}$ [107] while a double blackbody model suggests the upper bound $\sim 8 \times 10^{31} \mathrm{erg} / \mathrm{s}$ [108]. For J1308, the same models suggest $(3.3 \pm 0.5) \times 10^{32} \mathrm{erg} / \mathrm{s}$ and $2.6 \times 10^{32} \mathrm{erg} / \mathrm{s}$, respectively [109]. For J0720, both types of models give similar luminosities $\sim 2 \times 10^{32} \mathrm{erg} / \mathrm{s}$ [104]. A double blackbody fit yields the luminosity $(4 \pm 1) \times$ $10^{32} \mathrm{erg} / \mathrm{s}$ for J1605, which we adopt in our analysis [110]. The J0659 luminosity was determined with a double blackbody model including a power law, since it emits nonthermally in hard $\mathrm{x}$ rays as it is a pulsar [111]. We assume Gaussian priors on the NS luminosities and ages that include all measurements at $1 \sigma$. Note that the M7 have previously been the subject of searches for axion-induced hard $\mathrm{x}$-ray emission $[112,113]$.

In this Letter, we build off of the one-dimensional NS cooling code NSCOOL [114] to simulate NS cooling curves with axion energy losses. NSCOOL solves the energy balance and transport equations in full general relativity in the core and crust of the NS. An envelope model $T_{s}\left(T_{b}\right)$ that relates the interior and surface temperatures, $T_{b}$ and $T_{s}$, respectively, is glued to the exterior of the crust. After 


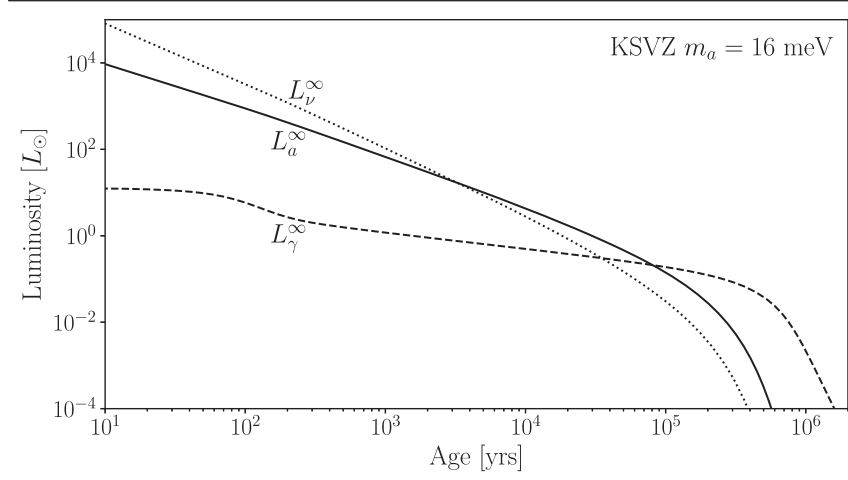

FIG. 2. The luminosity production from neutrinos, axions, and surface radiation for an example NS with the KSVZ axion at $m_{a}=16 \mathrm{meV}$. The NS parameters have been chosen to be those found in the profile likelihood procedure for J1605 with this axion mass: the BSk22 EOS, SBF-0-0 superfluidity model, $M_{\mathrm{NS}}=1.0 M_{\odot}$, and $\Delta M / M_{\odot}=10^{-12}$.

thermal relaxation, so that the NS has a uniform core temperature, integrating the energy balance equation over the interior of the NS leads to the cooling equation

$$
L_{\gamma}^{\infty}=-C \frac{d T_{b}^{\infty}}{d t}-L_{\nu}^{\infty}-L_{a}^{\infty}+H,
$$

where $L_{\gamma}^{\infty}=4 \pi R_{*, \infty}^{2}\left(T_{s}^{\infty}\right)^{4}$ is the photon luminosity, and $t$ is time. (Throughout this Letter, the infinity superscript will indicate that the value is taken to be that as measured by a distant observer.) The heat capacity of the NS is $C, L_{\nu}^{\infty}$ is the neutrino luminosity, $L_{a}^{\infty}$ is the axion luminosity, and $H$ accounts for possible heating sources, such as from magnetic field decay (see Fig. 2 for an illustration). Note that we include important corrections to the neutrino emissivities relative to those in NSCOOL [114], which we discuss shortly, and we also assume that $H=0$, since magnetic field induced heating likely plays a subdominant role in constraining $L_{a}^{\infty}$ (see the SM [60]). The solution of this equation yields the NS cooling curve $L_{\gamma}^{\infty}(t, \boldsymbol{\theta})$, where $\boldsymbol{\theta}$ parametrizes the particular choices of axion and NS properties. The axion is parametrized by its mass $m_{a}$ and coupling constants to nucleons, while for the NS, we need to know (i) the NS mass $M_{\mathrm{NS}}$, (ii) the EOS, (iii) the superfluidity model $\Delta\left(T_{b}\right)$, and (iv) the envelope model parametrized by the mass of light elements $\Delta M$.

The axion energy losses from nucleon scattering processes are determined by the axion-neutron and axionproton dimensionless coupling constants $C_{p}$ and $C_{n}$, respectively, in addition to $f_{a}$; the axion-nucleon interactions are of the form $\mathcal{L} \supset\left(C_{N} / 2 f_{a}\right) \bar{\psi}_{N} \gamma^{\mu} \gamma_{5} \psi_{N} \partial_{\mu} a$ with $N=p, n, \psi_{N}$ the nucleon fields, and $a$ the axion field. In the KSVZ axion model, $C_{p}=-0.47 \pm 0.03$ and $C_{n}=$ $-0.02 \pm 0.03$ [9], while in the DFSZ model $C_{p}$ and $C_{n}$ are functions of $\tan \beta$, which is the ratio of the vacuum expectation values of the up- to down-type Higgs doublets in that theory: $C_{n}=(-0.160 \pm 0.025)+0.414 \sin ^{2} \beta, C_{p}=$ $(-0.182 \pm 0.025)-0.435 \sin ^{2} \beta$ [9]. Additional axion models are also possible [115], for which it is useful to define the dimensionless coupling constants $g_{a N N}=$ $C_{N} m_{N} / f_{a}$, with $m_{N}$ the nucleon mass. Note that the uncertainties on the KSVZ and DFSZ axion couplings arise from lattice QCD [9]; to make contact with previous literature, we assume the central values.

When computing the axion luminosities, we account for axion bremsstrahlung [40,41] from nucleons and axion production from Cooper pair breaking and formation (PBF). If the NS core temperature is below the superfluid critical temperature, nucleons form Cooper pairs and condense into a superfluid phase. These Cooper pairs can liberate energy in the form of neutrinos [116,117] or axions [53,118] when breaking and forming. The PBF processes may dominate the axion luminosity at temperatures near the superfluid transition temperature, while the bremsstrahlung processes are exponentially suppressed at lower temperatures. To evaluate the axion and neutrino emission rates, for both $\mathrm{PBF}$ and bremsstrahlung production, we account for the medium-dependent axion-nucleon and pion-nucleon couplings $[119,120]$, which have not been included in earlier work on axion emission from compact stars or supernovae. See the SM [60] for details.

We make one additional modification to NSCOOL to help quantify the effects of astrophysical uncertainties. The addition of light elements (hydrogen, helium, and carbon) in the NS envelope changes the expected relation between the surface and core temperatures, which, in turn, affects the observed surface luminosity even for the same internal state. We incorporate the analytic formulae in Ref. [121] into NSCOOL in order to cool a NS with a mass $\Delta M$ of light elements layered on top of the default iron surface. Values for $\Delta M$ can span from $0 M_{\odot}$, such that the NS has a pure iron surface, to $\sim 10^{-7} M_{\odot}$, which is the mass of the entire envelope. In practice, we modify the equation $T_{s}\left(T_{b}\right)$ to account for the addition of light elements, which can change the photon luminosity of the NS by up to a factor $\sim 5$ before the photon cooling stage and $\gtrsim 100$ after. Since each $\Delta M$ value requires a dedicated NSCOOL simulation, we use a discrete number eight of equally log-spaced values for $\Delta M / M_{\odot}$ ranging from $10^{-20} M_{\odot}$ to $10^{-6} M_{\odot}$. Similarly, we discretize the NS mass range with six equally spaced masses between $1 M_{\odot}$ and $2 M_{\odot}$.

We simulate NSs for five distinct EOSs: APR [122], BSk22, BSk24, BSk25, and BSk26 [123]. The APR EOS is constructed using variational methods to model the twonucleon interaction incorporating the effects of many-body interactions and with the input of nucleon-nucleon scattering data. The BSk family of EOSs are generated by fitting the Skyrme effective interaction to atomic mass data. The distinct BSk EOSs are constructed with different assumed values of the Skyrme symmetry energy. These EOSs phenomenologically characterize the range of possible 
stiffnesses of the EOSs. Recently, data from the NICER telescope has allowed for the simultaneous measurements of the mass and radius of two NSs, PSR J0030 [124] and PSR J0740 [125], which can be used in conjunction with gravitational wave observations of NS mergers to constrain the EOS [125]. As we show in SM [60] Fig. S3, only the BSk22, BSk24, and BSk25 EOSs are consistent with the mass-radius data to within $1-2 \sigma$ significance. Thus, we restrict ourselves to this set of EOSs in the main Letter.

We consider three distinct superfluidity models, denoted in NSCOOL and here as 0-0-0, SFB-0-0, and SFB-0-T73. The first model assumes no superfluidity by setting the gaps to zero. The second model turns on the ${ }^{1} S_{0}$ neutron pairing gap from [126], and the third model additionally turns on the ${ }^{1} S_{0}$ proton pairing gap from [127]. Neutron ${ }^{3} P_{2}{ }^{3}{ }^{3} F_{2}$ pairing may also be possible (we will refer to this as ${ }^{3} P_{2}$ for brevity), but the estimate of this gap is more complicated, in part, because it appears at higher density where many-body interactions are more important (see, e.g., Ref. [128]).

Data analysis and results.-Given the set of cooling curves, we can compare them to the observed data in Table I. For a given QCD axion model, under the assumption of a particular NS EOS and superfluidity model $\Delta\left(T_{b}\right)$, let us label the present-day luminosity of a NS by $L\left(m_{a}, \boldsymbol{\theta}\right)$. The luminosity of NS $i$ is, then, jointly determined by the axion mass $m_{a}$ and the nuisance parameters $\boldsymbol{\theta}^{i}=\left\{M_{\mathrm{NS}}^{i}, \Delta M^{i}, t^{i}\right\}$ that characterize the NS. Now, we can write the likelihood for a single NS $i$ as

$$
\begin{aligned}
\mathcal{L}_{i}\left(\boldsymbol{d}_{i} \mid m_{a}, \boldsymbol{\theta}^{i}\right)= & \mathcal{N}\left(L\left(m_{a}, \boldsymbol{\theta}^{i}\right)-L_{0}^{i}, \sigma_{L}^{i}\right) \\
& \times \mathcal{N}\left(t^{i}-t_{0}^{i}, \sigma_{t}^{i}\right),
\end{aligned}
$$

where we have introduced the NS data set $\boldsymbol{d}_{i}=$ $\left\{L_{0}^{i}, \sigma_{L}^{i}, t_{0}^{i}, \sigma_{t}^{i}\right\}$, where $L_{0}^{i}$ is the measured luminosity of the NS with uncertainty $\sigma_{L}^{i}$. Similarly, $t_{0}^{i}$ is the measured age of the NS with uncertainty $\sigma_{t}^{i}$. The probability of observing a value $x$ under the zero-mean Gaussian distribution with standard deviation $\sigma$ is denoted by $\mathcal{N}(x, \sigma)$. The joint likelihood $\mathcal{L}\left(\boldsymbol{d} \mid m_{a}, \boldsymbol{\theta}\right)$ over all five NSs is constructed by taking the product of (2) over the NSs. Note that the total list of model parameters is denoted by $\boldsymbol{\theta}=\left\{\boldsymbol{\theta}^{i}\right\}_{i=1}^{5}$. The best-fit axion mass $\hat{m}_{a}$ and nuisance parameters $\hat{\boldsymbol{\theta}}$ can be determined for a given choice of EOS and superfluidity model by maximizing the joint likelihood. To test for systematic mismodeling, we allow $m_{a}<0$, with the axion luminosity multiplied by $\operatorname{sign}\left(m_{a}\right)$.

Additionally, given the large number of nuisance parameters, many of which have nontrivial degeneracy with the signal parameter $m_{a}$, we determine the $95 \%$ upper limit on $m_{a}$, defined by $m_{a}^{95}$, by the Neyman construction of the 95\% confidence interval for $m_{a}$ through a Monte Carlo (MC) procedure rather than by invoking Wilks' theorem.
TABLE I. The properties of the NSs considered in this LetterRX J1856.6 - 3754, RX J1308.6 + 2127, RX J0720.4 - 3125, RX J1605.3 + 3249, PSR J0659 + 1414-which we abbreviate throughout this Letter. We include all known NSs with ages above $10^{5}$ years and robust age and luminosity measurements (see, e.g., Ref. [58]). Younger NSs are discussed in the SM [60].

\begin{tabular}{lccc}
\hline \hline Name & $L_{\gamma}^{\infty}\left(10^{33} \mathrm{erg} / \mathrm{s}\right)$ & Age $(\mathrm{Myr})$ & References \\
\hline $\mathrm{J} 1856$ & $0.065 \pm 0.015$ & $0.42 \pm 0.08$ & {$[102,107,108]$} \\
$\mathrm{J} 1308$ & $0.32 \pm 0.06$ & $0.55 \pm 0.25$ & {$[103,109]$} \\
$\mathrm{J} 0720$ & $0.22 \pm 0.11$ & $0.85 \pm 0.15$ & {$[104,129]$} \\
$\mathrm{J} 1605$ & $0.4 \pm 0.1$ & $0.44 \pm 0.07$ & {$[105,110]$} \\
J0659 & $0.28 \pm 0.14$ & $0.35 \pm 0.044$ & {$[59,111]$} \\
\hline \hline
\end{tabular}

Similarly, we determine the significance of the axion model over the null hypothesis through MC simulations of the null hypothesis, instead of relying on Wilks' theorem. (See the SM [60] for details.)

We choose the $95 \%$ upper limit over the ensemble of nine EOS and superfluidity combinations that gives the most conservative limit. For the KSVZ axion model, we find that $m_{a}^{95} \approx 16 \mathrm{meV}$ with the BSk22 EOS model and the SFB-0-0 superfluidity model; the strongest constraint over all combinations is $m_{a}^{95} \approx 6 \mathrm{meV}$ with the BSk25 EOS and the SFB-0-T73 model. With that said, the SFB-0-T73 model is the worst fit to the data, with the best-fit axion mass being negative at $\sim 1.6 \sigma$ significance. The best-fitting model is that with the BSk22 EOS and no superfluidity, for which the limit is $m_{a}^{95} \approx 14 \mathrm{meV}$ and the best-fit axion mass being negative at $\sim 0.36 \sigma$. From these results, we conclude that the NS cooling data show no evidence for the KSVZ axion.

For the DFSZ axion, the results depend on the value of $\tan \beta$. In Fig. 3, we show $m_{a}^{95}$ as a function of $\tan \beta$, with the shaded band showing the range of limits found over all EOS and superfluidity combinations. The weakest limit (bold) is achieved for all $\tan \beta$ for the no superfluidity model with the BSk22 EOS. We compare these upper limits to those from horizontal branch (HB) $[130,131]$, red giant branch (RGB) [132,133], and SN 1987A [45] cooling. Note, however, that the SN 1987A limit is approximate, since, e.g., it arises from the rough requirement $L_{a}^{\infty}<L_{\nu}^{\infty}$ for the proto-NS, and also, it does not account for the density-dependent couplings for axions and neutrinos, which we estimate should weaken the SN 1987A limit by a factor $\sim 1.3-1.6$, depending on the EOS. We also show the projected discovery reach for the future IAXO experiment [33]; our results leave open a narrow mass range $\sim 10 \mathrm{meV}$ where IAXO may discover the QCD axion. In the axion model with only an axion-neutron (axion-proton) coupling, we constrain $\left|g_{\text {ann }}\right|<1.3 \times 10^{-9}$ $\left(\left|g_{\text {app }}\right|<1.5 \times 10^{-9}\right)$ at $95 \%$ confidence. 


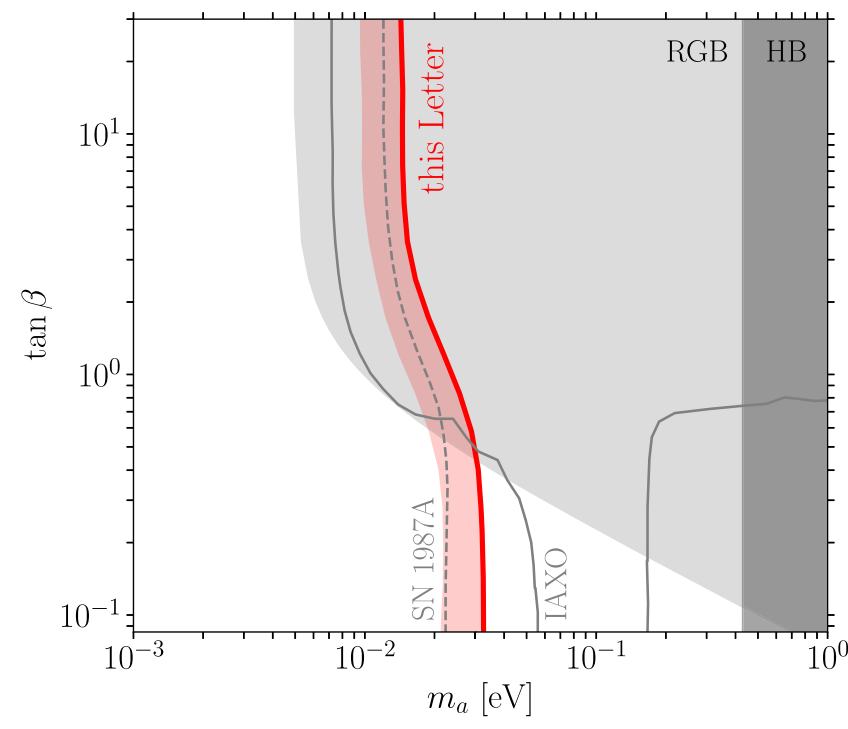

FIG. 3. Upper limit from this Letter on the DFSZ axion mass $m_{a}$ as a function of $\tan \beta$, which controls the relative coupling of the axion to neutrons and protons. The width of the shaded red band reflects the uncertainty on the upper limit by varying over superfluidity and EOS models. We compare our upper limits to existing constraints and the projected IAXO discovery sensitivity.

Discussion.-In this Letter, we present a search for the QCD axion from NS cooling, comparing NS cooling simulations with axions to luminosity and kinematically determined age data from five NSs. The NSs that are most important for our analysis are $\mathrm{J} 0720$, J1605, and $\mathrm{J} 1308$, as further highlighted in the SM [60].

Our upper limits disfavor at $95 \%$ QCD axions with masses $m_{a} \gtrsim 10-30 \mathrm{meV}$, depending on the axion model, which constrains the axion interpretation of the previously observed stellar cooling anomalies [134,135]. The limits may be stronger if ${ }^{3} P_{2}$ superfluidity is active in the NS cores, as we discuss in the SM [60], though large ${ }^{3} P_{2}$ gaps appear disfavored by the isolated NS data. Many-body nuclear techniques should provide improved estimates of the energy gaps of the ${ }^{1} S_{0}$ (neutron), ${ }^{1} S_{0}$ (proton), and ${ }^{3} P_{2}$ (neutron) pairings in the future [128]. On the other hand, more work should be done to rigorously assess the possible impact of heating mechanisms such as magnetic field decay on the axion limits, for example, using fully self-consistent simulations along the lines of those in Ref. [136,137]. Axions may also be produced from more exotic forms of matter, such as hyperon superfluids and pionic and kaonic Bose Einstein condensates, and these channels should be investigated as the NS EOS and composition becomes better understood.

We thank K. Cranmer, S. McDermott, T. Opferkuch, G. Raffelt, and S. Witte for useful discussions and T. Opferkuch for sharing data related to the EOS. M. B. was supported by the DOE under Award No. DESC0007968.
A. J. L. was supported in part by the National Science Foundation under Grant No. 2114024. C. D. and B. R. S. were supported in part by the DOE Early Career Grant No. DESC0019225. J. F. was supported by a Pappalardo Fellowship. This research used resources from the Lawrencium computational cluster provided by the IT Division at the Lawrence Berkeley National Laboratory, supported by the Director, Office of Science, and Office of Basic Energy Sciences, of the U.S. Department of Energy under Contract No. DE-AC02-05CH11231.

[1] R. D. Peccei and Helen R. Quinn, CP Conservation in the Presence of Instantons, Phys. Rev. Lett. 38, 1440 (1977).

[2] R. D. Peccei and Helen R. Quinn, Constraints imposed by CP conservation in the presence of instantons, Phys. Rev. D 16, 1791 (1977).

[3] Steven Weinberg, A New Light Boson?, Phys. Rev. Lett. 40, 223 (1978).

[4] Frank Wilczek, Problem of Strong p and t Invariance in the Presence of Instantons, Phys. Rev. Lett. 40, 279 (1978).

[5] John Preskill, Mark B. Wise, and Frank Wilczek, Cosmology of the invisible axion, Phys. Lett. 120B, 127 (1983).

[6] L. F. Abbott and P. Sikivie, A cosmological bound on the invisible axion, Phys. Lett. 120B, 133 (1983).

[7] Michael Dine and Willy Fischler, The not so harmless axion, Phys. Lett. 120B, 137 (1983).

[8] Pierre Sikivie, Invisible axion search methods, Rev. Mod. Phys. 93, 015004 (2021).

[9] Giovanni Grilli di Cortona, Edward Hardy, Javier Pardo Vega, and Giovanni Villadoro, The QCD axion, precisely, J. High Energy Phys. 01 (2016) 034.

[10] Asimina Arvanitaki, Savas Dimopoulos, Sergei Dubovsky, Nemanja Kaloper, and John March-Russell, String axiverse, Phys. Rev. D 81, 123530 (2010).

[11] Asimina Arvanitaki and Sergei Dubovsky, Exploring the string axiverse with precision black hole physics, Phys. Rev. D 83, 044026 (2011).

[12] Vitor Cardoso, Óscar J.C. Dias, Gavin S. Hartnett, Matthew Middleton, Paolo Pani, and Jorge E. Santos, Constraining the mass of dark photons and axion-like particles through black-hole superradiance, J. Cosmol. Astropart. Phys. 03 (2018) 043.

[13] Michael Dine, Willy Fischler, and Mark Srednicki, A simple solution to the strong CP problem with a harmless axion, Phys. Lett. 104B, 199 (1981).

[14] A. R. Zhitnitsky, On possible suppression of the axion hadron interactions. (In Russian), Sov. J. Nucl. Phys. 31, 260 (1980).

[15] N. Du et al. (ADMX Collaboration), A Search for Invisible Axion Dark Matter with the Axion Dark Matter Experiment, Phys. Rev. Lett. 120, 151301 (2018).

[16] T. Braine et al. (ADMX Collaboration), Extended Search for the Invisible Axion with the Axion Dark Matter Experiment, Phys. Rev. Lett. 124, 101303 (2020).

[17] C. Bartram et al. (ADMX Collaboration), Search for Invisible Axion Dark Matter in the 3.3-4.2 $\mu \mathrm{eV}$ Mass Range, Phys. Rev. Lett. 127, 261803 (2021). 
[18] S. Asztalos et al. (ADMX Collaboration), Large scale microwave cavity search for dark matter axions, Phys. Rev. D 64, 092003 (2001).

[19] K. M. Backes et al. (HAYSTAC Collaboration), A quantum-enhanced search for dark matter axions, Nature (London) 590, 238 (2021).

[20] Jihn E. Kim, Weak Interaction Singlet and Strong CP Invariance, Phys. Rev. Lett. 43, 103 (1979).

[21] Mikhail A. Shifman, A. I. Vainshtein, and Valentin I. Zakharov, Can confinement ensure natural CP invariance of strong interactions?, Nucl. Phys. B166, 493 (1980).

[22] Yonatan Kahn, Benjamin R. Safdi, and Jesse Thaler, Broadband and Resonant Approaches to Axion Dark Matter Detection, Phys. Rev. Lett. 117, 141801 (2016).

[23] J. L. Ouellet, C. P. Salemi, J. W. Foster, R. Henning, Z. Bogorad, J. M. Conrad, J. A. Formaggio, Y. Kahn, J. Minervini, A. Radovinsky, N. L. Rodd, B. R. Safdi, J. Thaler, D. Winklehner, and L. Winslow et al., First Results from ABRACADABRA-10 cm: A Search for Sub- $\mu \mathrm{eV}$ Axion Dark Matter, Phys. Rev. Lett. 122, 121802 (2019).

[24] C. P. Salemi, J. W. Foster, J. L. Ouellet, A. Gavin, K. M. W. Pappas, S. Cheng, K. A. Richardson, R. Henning, Y. Kahn, R. Nguyen, N. L. Rodd, B. R. Safdi, and L. Winslow, Search for Low-Mass Axion Dark Matter with ABRACADABRA-10 cm, Phys. Rev. Lett. 127, 081801 (2021).

[25] Maximiliano Silva-Feaver et al., Design overview of DM radio pathfinder experiment, IEEE Trans. Appl. Supercond. 27, 1 (2017).

[26] Dmitry Budker, Peter W. Graham, Micah Ledbetter, Surjeet Rajendran, and Alex Sushkov, Proposal for a Cosmic Axion Spin Precession Experiment (CASPEr), Phys. Rev. X 4, 021030 (2014).

[27] Antoine Garcon et al., The Cosmic Axion Spin Precession Experiment (CASPEr): A dark-matter search with nuclear magnetic resonance, Quantum Sci. Technol. 3, 014008 (2018).

[28] D. Aybas et al., Search for Axionlike Dark Matter Using Solid-State Nuclear Magnetic Resonance, Phys. Rev. Lett. 126, 141802 (2021).

[29] P. Brun et al. (MADMAX Collaboration), A new experimental approach to probe QCD axion dark matter in the mass range above $40 \mu \mathrm{eV}$, Eur. Phys. J. C 79, 186 (2019).

[30] Matthew Lawson, Alexander J. Millar, Matteo Pancaldi, Edoardo Vitagliano, and Frank Wilczek, Tunable Axion Plasma Haloscopes, Phys. Rev. Lett. 123, 141802 (2019).

[31] Asimina Arvanitaki and Andrew A. Geraci, Resonantly Detecting Axion-Mediated Forces with Nuclear Magnetic Resonance, Phys. Rev. Lett. 113, 161801 (2014).

[32] A. A. Geraci, G. Rybka, and K. van Bibber (ARIADNE Collaboration), Progress on the ARIADNE axion experiment, Springer Proc. Phys. 211, 151 (2018).

[33] E. Armengaud et al. (IAXO Collaboration), Physics potential of the International Axion Observatory (IAXO), J. Cosmol. Astropart. Phys. 06 (2019) 047.

[34] A. Abeln et al. (IAXO Collaboration), Conceptual design of BabyIAXO, the intermediate stage towards the International Axion Observatory, J. High Energy Phys. 05 (2021) 137.

[35] Jan Schütte-Engel, David J. E. Marsh, Alexander J. Millar, Akihiko Sekine, Francesca Chadha-Day, Sebastian Hoof,
Mazhar N. Ali, Kin-Chung Fong, Edward Hardy, and Libor Šmejkal, Axion quasiparticles for axion dark matter detection, J. Cosmol. Astropart. Phys. 08 (2021) 066.

[36] Jesse Liu et al. (BREAD Collaboration), Broadband solenoidal haloscope for terahertz axion detection, arXiv: 2111.12103.

[37] Raymond T. Co, Lawrence J. Hall, Keisuke Harigaya, Keith A. Olive, and Sarunas Verner, Axion kinetic misalignment and parametric resonance from inflation, J. Cosmol. Astropart. Phys. 08 (2020) 036.

[38] Marco Gorghetto, Edward Hardy, and Giovanni Villadoro, More axions from strings, SciPost Phys. 10, 050 (2021).

[39] D. G. Yakovlev, A. D. Kaminker, Oleg Y. Gnedin, and P. Haensel, Neutrino emission from neutron stars, Phys. Rep. 354, 1 (2001).

[40] N. Iwamoto, Axion Emission from Neutron Stars, Phys. Rev. Lett. 53, 1198 (1984).

[41] Naoki Iwamoto, Nucleon-nucleon bremsstrahlung of axions and pseudoscalar particles from neutron star matter, Phys. Rev. D 64, 043002 (2001).

[42] Georg G. Raffelt, Astrophysical axion bounds, Lect. Notes Phys. 741, 51 (2008).

[43] Tobias Fischer, Sovan Chakraborty, Maurizio Giannotti, Alessandro Mirizzi, Alexandre Payez, and Andreas Ringwald, Probing axions with the neutrino signal from the next galactic supernova, Phys. Rev. D 94, 085012 (2016).

[44] Jae Hyeok Chang, Rouven Essig, and Samuel D. McDermott, Supernova 1987A constraints on Sub-GeV dark sectors, millicharged particles, the QCD axion, and an axion-like particle, J. High Energy Phys. 09 (2018) 051.

[45] Pierluca Carenza, Tobias Fischer, Maurizio Giannotti, Gang Guo, Gabriel Martínez-Pinedo, and Alessandro Mirizzi, Improved axion emissivity from a supernova via nucleon-nucleon bremsstrahlung, J. Cosmol. Astropart. Phys. 10 (2019) 016; 05 (2020) E01.

[46] Pierluca Carenza, Bryce Fore, Maurizio Giannotti, Alessandro Mirizzi, and Sanjay Reddy, Enhanced Supernova Axion Emission and its Implications, Phys. Rev. Lett. 126, 071102 (2021).

[47] Dany Page, Madappa Prakash, James M. Lattimer, and Andrew W. Steiner, Rapid Cooling of the Neutron Star in Cassiopeia A Triggered by Neutron Superfluidity in Dense Matter, Phys. Rev. Lett. 106, 081101 (2011).

[48] Peter S. Shternin, Dmitry G. Yakovlev, Craig O. Heinke, Wynn C. G. Ho, and Daniel J. Patnaude, Cooling neutron star in the Cassiopeia A supernova remnant: Evidence for superfluidity in the core, Mon. Not. R. Astron. Soc. 412, L108 (2011).

[49] Lev B. Leinson, Superfluid phases of triplet pairing and rapid cooling of the neutron star in Cassiopeia A, Phys. Lett. B 741, 87 (2015).

[50] L. B. Leinson, Axion mass limit from observations of the neutron star in Cassiopeia A, J. Cosmol. Astropart. Phys. 08 (2014) 031.

[51] Koichi Hamaguchi, Natsumi Nagata, Keisuke Yanagi, and Jiaming Zheng, Limit on the axion decay constant from the cooling neutron star in Cassiopeia A, Phys. Rev. D 98, 103015 (2018). 
[52] Lev B. Leinson, Impact of axions on the Cassiopea A neutron star cooling, J. Cosmol. Astropart. Phys. 09 (2021) 001.

[53] Armen Sedrakian, Axion cooling of neutron stars, Phys. Rev. D 93, 065044 (2016).

[54] Armen Sedrakian, Axion cooling of neutron stars. II. Beyond hadronic axions, Phys. Rev. D 99, 043011 (2019).

[55] Nitsan Bar, Kfir Blum, and Guido D'Amico, Is there a supernova bound on axions?, Phys. Rev. D 101, 123025 (2020).

[56] Dany Page, Mikhail V. Beznogov, Iván Garibay, James M. Lattimer, Madappa Prakash, and Hans-Thomas Janka, NS 1987A in SN 1987A, Astrophys. J. 898, 125 (2020).

[57] B. Posselt and G. G. Pavlov, Upper limits on the rapid cooling of the Central Compact Object in Cas A, Astrophys. J. 864, 135 (2018).

[58] A. Y. Potekhin, D. A. Zyuzin, D. G. Yakovlev, M. V. Beznogov, and Yu. A. Shibanov, Thermal luminosities of cooling neutron stars, Mon. Not. R. Astron. Soc. 496, 5052 (2020).

[59] Hiromasa Suzuki, Aya Bamba, and Shinpei Shibata, Quantitative age estimation of supernova remnants and associated pulsars, Astrophys. J. 914, 103 (2021).

[60] See Supplemental Material at http://link.aps.org/ supplemental/10.1103/PhysRevLett.128.091102 for calculations of the axion and neutrino emissivities, our statistical methodology, extended results for the analyses mentioned in the main body, and our estimates for the effects of magnetic field decay on the axion upper limits, which includes Refs. [61-101].

[61] B. L. Friman and O. V. Maxwell, Neutron star neutrino emissivities, Astrophys. J. 232, 541 (1979).

[62] D. G. Yakovlev and K. P. Levenfish, Modified URCA process in neutron star cores, Astron. Astrophys. 297, 717 (1995).

[63] Ron Mayle, James R. Wilson, John R. Ellis, Keith A. Olive, David N. Schramm, and Gary Steigman, Updated constraints on axions from SN 1987a, Phys. Lett. B 219, 515 (1989).

[64] K. P. Levenfish and D. G. Yakovlev, Suppression of neutrino energy losses in reactions of direct urca processes by superfluidity in neutron star nuclei, Astron. Lett. 20, 43 (1994).

[65] D. G. Yakovlev, A. D. Kaminker, and K. P. Levenfish, Neutrino emission due to Cooper pairing of nucleons in cooling neutron stars, Astron. Astrophys. 343, 650 (1999).

[66] Brynmor Haskell and Armen Sedrakian, Superfluidity and superconductivity in neutron stars, Astrophysics and Space Science Library 457, 401 (2018).

[67] K. P. Levenfish and D. G. Yakovlev, Specific heat of neutron star cores with superfluid nucleons, Astronomy Reports 38, 247 (1994).

[68] Dany Page, James M. Lattimer, Madappa Prakash, and Andrew W. Steiner, Minimal cooling of neutron stars: A new paradigm, Astrophys. J. Suppl. Ser. 155, 623 (2004).

[69] K. P. Levenfish and D. G. Yakovlev, Standard and enhanced cooling of neutron stars with superfluid cores, Astron. Lett. 22, 49 (1996).
[70] Dany Page, James M. Lattimer, Madappa Prakash, and Andrew W. Steiner, Neutrino emission from Cooper pairs and minimal cooling of neutron stars, Astrophys. J. 707, 1131 (2009).

[71] Glen Cowan, Kyle Cranmer, Eilam Gross, and Ofer Vitells, Asymptotic formulae for likelihood-based tests of new physics, Eur. Phys. J. C 71, 1554 (2011); 73, 2501(E) (2013).

[72] W. B. Ashworth, Jr., A Probable Flamsteed Observation of the Cassiopeia A Supernova, Journal for the history of astronomy 11, 1 (1980).

[73] Robert A. Fesen, Molly C. Hammell, Jon Morse, Roger A. Chevalier, Kazimierz J. Borkowski, Michael A. Dopita, Christopher L. Gerardy, Stephen S. Lawrence, John C. Raymond, and Sidney van den Bergh, The expansion asymmetry and age of the Cassiopeia A supernova remnant, Astrophys. J. 645, 283 (2006).

[74] D. Klochkov, V. Suleimanov, G. Pühlhofer, D. G. Yakovlev, A. Santangelo, and K. Werner, The neutron star in HESSJ1731-347: Central compact objects as laboratories to study the equation of state of superdense matter, Astron. Astrophys. 573, A53 (2015).

[75] F. Acero, M. Lemoine-Goumard, M. Renaud, J. Ballet, J. W. Hewitt, R. Rousseau, and T. Tanaka, Study of TeV shell supernova remnants at gamma-ray energies, Astron. Astrophys. 580, A74 (2015).

[76] Y. Cui, G. Pühlhofer, and A. Santangelo, A young supernova remnant illuminating nearby molecular clouds with cosmic rays, Astron. Astrophys. 591, A68 (2016).

[77] Nigel Maxted, Michael Burton, Catherine Braiding, Gavin Rowell, Hidetoshi Sano, Fabien Voisin, Massimo Capasso, Gerd Pühlhofer, and Yasuo Fukui, Probing the local environment of the supernova remnant HESS J1731 347 with CO and CS observations, Mon. Not. R. Astron. Soc. 474, 662 (2018).

[78] Wynn C. G. Ho, Yue Zhao, Craig O. Heinke, D. L. Kaplan, Peter S. Shternin, and M. J. P. Wijngaarden, X-ray bounds on cooling, composition, and magnetic field of the Cassiopeia A neutron star and young central compact objects, Mon. Not. R. Astron. Soc. 506, 5015 (2021).

[79] I. Lovchinsky, P. Slane, B. M. Gaensler, J. P. Hughes, C. Y. Ng, J. S. Lazendic, J. D. Gelfand, and C. L. Brogan, A Chandra observation of supernova remnant G350.1-0.3 and its central compact object, Astrophys. J. 731, 70 (2011).

[80] Martin G. F. Mayer and Werner Becker, A kinematic study of central compact objects and their host supernova remnants, Astron. Astrophys. 651, A40 (2021).

[81] Aida Kirichenko, Andrey Danilenko, Yury Shibanov, Peter Shternin, Sergey Zharikov, and Dmitry Zyuzin, Deep optical observations of the $\gamma$-ray pulsar $\mathrm{J} 0357+3205$, Astron. Astrophys. 564, A81 (2014).

[82] Chi-Y. Ng, Roger W. Romani, Walter F. Brisken, Shami Chatterjee, and Michael Kramer, The origin and motion of PSR J0538 + 2817 in S147, Astrophys. J. 654, 487 (2007).

[83] Craig O. Heinke and Wynn C. G. Ho, Direct observation of the cooling of the Cassiopeia a neutron star, Astrophys. J. Lett. 719, L167 (2010). 
[84] K. G. Elshamouty, C. O. Heinke, G. R. Sivakoff, W. C. G. Ho, P. S. Shternin, D. G. Yakovlev, D. J. Patnaude, and L. David, Measuring the cooling of the neutron star in Cassiopeia A with all Chandra X-ray observatory detectors, Astrophys. J. 777, 22 (2013).

[85] Mikhail V. Beznogov, Ermal Rrapaj, Dany Page, and Sanjay Reddy, Constraints on axion-like particles and nucleon pairing in dense matter from the hot neutron star in HESS J1731 - 347, Phys. Rev. C 98, 035802 (2018).

[86] Lev B. Leinson, Constraints on axions from neutron star in HESS J1731 - 347, J. Cosmol. Astropart. Phys. 11 (2019) 031.

[87] W. W. Tian, D. A. Leahy, M. Haverkorn, and B. Jiang, Discovery of the counterpart of TeV Gamma-ray source HESS J1731-347: A new SNR G353.6-0.7 with radio and X-ray images, Astrophys. J. Lett. 679, L85 (2008).

[88] D. Ding, A. Rios, H. Dussan, W. H. Dickhoff, S. J. Witte, A. Carbone, and A. Polls, Pairing in high-density neutron matter including short- and long-range correlations, Phys. Rev. C 94, 025802 (2016); 94, 029901(E) (2016).

[89] Shao-Peng Tang, Jin-Liang Jiang, Wei-Hong Gao, Yi-Zhong Fan, and Da-Ming Wei, The masses of isolated neutron stars inferred from the gravitational redshift measurements, Astrophys. J. 888, 45 (2020).

[90] P. Haensel, V. A. Urpin, and D. G. Yakovlev, Ohmic decay of internal magnetic fields in neutron stars, Astron. Astrophys. 229, 133 (1990).

[91] Juan A. Miralles, Vadim Urpin, and Denis Konenkov, Joule heating and the thermal evolution of old neutron stars, Astrophys. J. 503, 368 (1998).

[92] D. Page, U. Geppert, and T. Zannias, General relativistic treatment of the thermal, magnetic and rotational evolution of neutron stars with crustal magnetic fields, Astron. Astrophys. 360, 1052 (2000).

[93] U. Geppert, M. Rheinhardt, and J. Gil, Spot-like structures of neutron star surface magnetic fields, Astron. Astrophys. 412, L33 (2003).

[94] P. Arras, A. Cumming, and Christopher Thompson, Magnetars: Time evolution, superfluid properties, and mechanism of magnetic field decay, Astrophys. J. Lett. 608, L49 (2004).

[95] Andrew Cumming, Phil Arras, and Ellen G. Zweibel, Magnetic field evolution in neutron star crusts due to the Hall effect and Ohmic decay, Astrophys. J. 609, 999 (2004).

[96] Jose A. Pons, Bennett Link, Juan A. Miralles, and Ulrich Geppert, Evidence for Heating of Neutron Stars by Magnetic Field Decay, Phys. Rev. Lett. 98, 071101 (2007).

[97] Jose A. Pons and U. Geppert, Magnetic field dissipation in neutron star crusts: From magnetars to isolated neutron stars, Astron. Astrophys. 470, 303 (2007).

[98] Deborah N. Aguilera, Jose A. Pons, and Juan A. Miralles, The impact of magnetic field on the thermal evolution of neutron stars, Astrophys. J. Lett. 673, L167 (2008).

[99] S. B. Popov, J. A. Pons, J. A. Miralles, P. A. Boldin, and B. Posselt, Population synthesis studies of isolated neutron stars with magnetic field decay, Mon. Not. R. Astron. Soc. 401, 2675 (2010).
[100] Daniele Viganò, Nanda Rea, Jose A. Pons, Rosalba Perna, Deborah N. Aguilera, and Juan A. Miralles, Unifying the observational diversity of isolated neutron stars via magneto-thermal evolution models, Mon. Not. R. Astron. Soc. 434, 123 (2013).

[101] Daniele Viganò, Alberto García-García, José A. Pons, Clara Dehman, and Vanessa Graber, Magneto-thermal evolution of neutron stars with coupled Ohmic, Hall and ambipolar effects via accurate finite-volume simulations, Comput. Phys. Commun. 265, 108001 (2021).

[102] R. P. Mignani, D. Vande Putte, M. Cropper, R. Turolla, S. Zane, L. J. Pellizza, L. A. Bignone, N. Sartore, and A. Treves, The birthplace and age of the isolated neutron star RX J1856.5-3754, Mon. Not. R. Astron. Soc. 429, 3517 (2013).

[103] C. Motch, A. M. Pires, F. Haberl, A. Schwope, and V. E. Zavlin, Proper motions of thermally emitting isolated neutron stars measured with Chandra, Astron. Astrophys. 497, 423 (2009).

[104] N. Tetzlaff, T. Eisenbeiss, R. Neuhäuser, and M. M. Hohle, The origin of RX J1856.5-3754 and RX J0720.4-3125updated using new parallax measurements, Mon. Not. R. Astron. Soc. 417, 617 (2011).

[105] N. Tetzlaff, J. G. Schmidt, M. M. Hohle, and R. Neuhäuser, Neutron stars from young nearby associations: The origin of RX J1605.3 + 3249, Pub. Astron. Soc. Aust. 29, 98 (2012).

[106] M. V. Beznogov, A. Y. Potekhin, and D. G. Yakovlev, Heat blanketing envelopes of neutron stars, Phys. Rep. 919, 1 (2021).

[107] Wynn C. G. Ho, David L. Kaplan, Philip Chang, Matthew van Adelsberg, and Alexander Y. Potekhin, Magnetic hydrogen atmosphere models and the neutron star RX J1856.5 - 3754, Mon. Not. R. Astron. Soc. 375, 821 (2007).

[108] N. Sartore, A. Tiengo, S. Mereghetti, A. De Luca, R. Turolla, and F. Haberl, Spectral monitoring of RX J1856.5 - 3754 with XMM-Newton. Analysis of EPICpn data, Astron. Astrophys. 541, A66 (2012).

[109] V. Hambaryan, V. Suleimanov, A. D. Schwope, R. Neuhäuser, K. Werner, and A. Y. Potekhin, Phase-resolved spectroscopic study of the isolated neutron star RBS 1223 (1RXS J130848.6 + 212708), Astron. Astrophys. 534, A74 (2011).

[110] A. M. Pires, A. D. Schwope, F. Haberl, V. E. Zavlin, C. Motch, and S. Zane, A deep XMM-Newton look on the thermally emitting isolated neutron star RX J1605.3 + 3249, Astron. Astrophys. 623, A73 (2019).

[111] S. Zharikov, D. Zyuzin, Yu. Shibanov, A. Kirichenko, R. E. Mennickent, S. Geier, and A. Cabrera-Lavers, PSR B0656 + 14: the unified outlook from the infrared to X rays, Mon. Not. R. Astron. Soc. 502, 2005 (2021).

[112] Christopher Dessert, Joshua W. Foster, and Benjamin R. Safdi, Hard x-ray excess from the magnificent seven neutron stars, Astrophys. J. 904, 42 (2020).

[113] Malte Buschmann, Raymond T. Co, Christopher Dessert, and Benjamin R. Safdi, Axion Emission Can Explain a New Hard X-Ray Excess from Nearby Isolated Neutron Stars, Phys. Rev. Lett. 126, 021102 (2021). 
[114] Dany Page, NSCool: Neutron star cooling code (2016), ascl:1609.009, http://ascl.net/1609.009.

[115] Luca Di Luzio, Maurizio Giannotti, Enrico Nardi, and Luca Visinelli, The landscape of QCD axion models, Phys. Rep. 870, 1 (2020).

[116] E. Flowers, M. Ruderman, and P. Sutherland, Neutrino pair emission from finite-temperature neutron superfluid and the cooling of young neutron stars, Astrophys. J. 205, 541 (1976).

[117] A. V. Senatorov and D. N. Voskresensky, Collective excitations in nucleonic matter and the problem of cooling of neutron stars, Phys. Lett. B 184, 119 (1987).

[118] Jochen Keller and Armen Sedrakian, Axions from cooling compact stars, Nucl. Phys. A897, 62 (2013).

[119] Tobias Fischer, The role of medium modifications for neutrino-pair processes from nucleon-nucleon bremsstrahlung: Impact on the protoneutron star deleptonization, Astron. Astrophys. 593, A103 (2016).

[120] Reuven Balkin, Javi Serra, Konstantin Springmann, and Andreas Weiler, The QCD axion at finite density, J. High Energy Phys. 07 (2020) 221.

[121] A. Y. Potekhin, G. Chabrier, and D. G. Yakovlev, Internal temperatures and cooling of neutron stars with accreted envelopes, Astron. Astrophys. 323, 415 (1997).

[122] A. Akmal, V. R. Pandharipande, and D. G. Ravenhall, Equation of state of nucleon matter and neutron star structure, Phys. Rev. C 58, 1804 (1998).

[123] J. M. Pearson, N. Chamel, A. Y. Potekhin, A. F. Fantina, C. Ducoin, A. K. Dutta, and S. Goriely, Unified equations of state for cold non-accreting neutron stars with BrusselsMontreal functionals. I. Role of symmetry energy, Mon. Not. R. Astron. Soc. 481, 2994 (2018); 486, 768(E) (2019).

[124] Thomas E. Riley et al., A NICER view of PSR $\mathrm{J} 0030+0451$ : Millisecond pulsar parameter estimation, Astrophys. J. Lett. 887, L21 (2019).

[125] M. C. Miller et al., The radius of PSR J0740 + 6620 from NICER and XMM-Newton data, Astrophys. J. Lett. 918, L28 (2021).

[126] Achim Schwenk, Bengt Friman, and Gerald E. Brown, Renormalization group approach to neutron matter: Quasiparticle interactions, superfluid gaps and the equation of state, Nucl. Phys. A713, 191 (2003).
[127] M. Baldo, J. Cugnon, A. Lejeune, and U. Lombardo, Proton and neutron superfluidity in neutron star matter, Nucl. Phys. A536, 349 (1992).

[128] Armen Sedrakian and John W. Clark, Superfluidity in nuclear systems and neutron stars, Eur. Phys. J. A 55, 167 (2019).

[129] V. Hambaryan, V. Suleimanov, F. Haberl, A. D. Schwope, R. Neuhäuser, M. Hohle, and K. Werner, The compactness of the isolated neutron star RX J0720.4 - 3125, Astron. Astrophys. 601, A108 (2017).

[130] Adrian Ayala, Inma Domínguez, Maurizio Giannotti, Alessandro Mirizzi, and Oscar Straniero, Revisiting the Bound on Axion-Photon Coupling from Globular Clusters, Phys. Rev. Lett. 113, 191302 (2014).

[131] Oscar Straniero, Adrian Ayala, Maurizio Giannotti, Alessandro Mirizzi, and Inma Dominguez, Axion-Photon Coupling: Astrophysical Constraints, in 11th Patras Workshop on Axions, WIMPs and WISPs (2015), pp. 77-81, 10.3204/DESY-PROC-2015-02/straniero_oscar.

[132] O. Straniero, C. Pallanca, E. Dalessandro, I. Dominguez, F. R. Ferraro, M. Giannotti, A. Mirizzi, and L. Piersanti, The RGB tip of galactic globular clusters and the revision of the axion-electron coupling bound, Astron. Astrophys. 644, A166 (2020).

[133] Francesco Capozzi and Georg Raffelt, Axion and neutrino bounds improved with new calibrations of the tip of the red-giant branch using geometric distance determinations, Phys. Rev. D 102, 083007 (2020).

[134] Maurizio Giannotti, Igor G. Irastorza, Javier Redondo, Andreas Ringwald, and Ken'ichi Saikawa, Stellar recipes for axion hunters, J. Cosmol. Astropart. Phys. 10 (2017) 010.

[135] Luca Di Luzio, Marco Fedele, Maurizio Giannotti, Federico Mescia, and Enrico Nardi, Stellar evolution confronts axion models, arXiv:2109.10368.

[136] Deborah N. Aguilera, Jose A. Pons, and Juan A. Miralles, 2D cooling of magnetized neutron stars, Astron. Astrophys. 486, 255 (2008).

[137] A. Y. Potekhin and G. Chabrier, Magnetic neutron star cooling and microphysics, Astron. Astrophys. 609, A74 (2018). 